Revista Internacional de Ciencias Podológicas

ISSN: 1989-5151

\title{
Relación entre el sexo, edad e índice de masa corporal y el Foot Function Index en pacientes con fascitis plantar
}

Fecha de recepción: 4 de abril de 2018 / Fecha de aceptación: 12 de septiembre de 2018

José Antonio García Vidal ${ }^{1}$, Aitor Baño Alcaraz², María Piedad Sánchez Martínez , Agustín Belmonte Albaladejo ${ }^{4}$, Rodrigo Martín San Agustín ${ }^{5}$

Resumen. Introducción: La fascitis plantar es una de las patologías de miembro inferior que más impacto causan en la persona produciendo dolor, discapacidad y limitación funcional. Una serie de factores incrementan el riesgo de sufrir fascitis plantar tales como predominio del sexo femenino, edad avanzada y un índice de masa corporal (IMC) elevado. El impacto que produce la fascitis plantar en la persona ser cuantificado por escalas para darnos información en la clínica acerca del estado de nuestros pacientes. Métodos y objetivos: Se realizó un estudio observacional transversal con 28 participantes que sufrían fascitis plantar y se les administra la encuesta FFI-sp con el propósito de determinar la relación entre las subescalas incluidas en el FFI-sp con el sexo, edad e IMC en pacientes con fascitis plantar. Resultados: No se han obtenido resultados significativos que indiquen relación del sexo, edad e IMC con las subescalas incluidas en el FFI-sp. Conclusión: No se ha encontrado relación entre FFI-sp y las variables sexo, edad e IMC.

Palabras clave: FFI-sp; sexo; edad; IMC; fascitis plantar.

[en] Relationship between sex, age and body mass index and the Foot Function Index in patients with plantar fasciitis

\begin{abstract}
Background: Plantar fasciitis is one of the lower limb pathologies that causes more impact on the person and it produces pain, disability and functional limitation. Some factors which increase the risk of plantar fasciitis such as female predominance, advanced age, and high body mass index (BMI). The impact of plantar fasciitis on people is quantified by scales to give us information in the clinic about the state of our patients. Methods and Objetives: A cross-sectional observational study was performed with 28 participants who are suffering plantar fasciitis and administered the FFI-sp in order to determine the relationship between the subcategories included in the FFI-sp with gender, age and BMI in patients with plantar fasciitis. Results: There have not been significant results indicating a relationship between gender, age and BMI with the subscales included in the FFI-sp. Conclusion: There's no relationship between gender, age and body mass index with the Foot Function Index in patients with plantar fasciitis.
\end{abstract}

Keywords: FFI-sp; gender; age; BMI; plantar fasciitis.

Sumario: 1. Introducción. 2. Objetivos. 3. Material y métodos. 4. Resultados. 5. Discusión. 6. Conclusión. 7. Bibliografía.

Cómo citar: García Vidal, J. A.; Baño Alcaraz, A. Sánchez Martínez, M. ${ }^{\text {a }}$.; Belmonte Albaladejo, A.; Martín San Agustín. R. (2018). Relación entre el sexo, edad e índice de masa corporal y el Foot Function Index en pacientes con fascitis plantar, en Revista internacional de ciencias podológicas 13 (1), 33-40.

\footnotetext{
Diplomado en Fisioterapia. Doctor por la Universidad de Murcia. Profesor del Departamento de Fisioterapia de la Universidad de Murcia. garciavidal@um.es

2 Diplomado en Fisioterapia. Doctor por la Universidad de Sevilla. Profesor del Departamento de Fisioterapia de la Universidad de Murcia. aitor.bano@um.es

3 Diplomado en Fisioterapia. Departamento de Fisioterapia de la Universidad de Murcia. mariapiedadsanchez@hotmail.com

4 4Dilomado en Podología y Fisioterapia. Departamento de Fisioterapia. Facultad de Fisioterapia. Universidad católica de Murcia (UCAM). agusbelmon@hotmail.com

5 5Diplomado en Fisiterapia. Departamento de Fisioterapia. Universidad de Valencia. rodrigo.martin@uv.es
} 


\section{Introducción}

Aproximadamente un $10 \%$ de la población ha sufrido o sufrirá dolor de pie a lo largo de su vida, en concreto, dolor en la fascia plantar, lo que denominamos como fascitis plantar ${ }^{1-5}$. La fascia plantar es el principal estabilizador estático y dinámico de los arcos longitudinales del pie, pero si la tensión supera los límites puede causar desgarros en la fascia plantar. Si esas tensiones son repetidas empezaran a causar en la fascia inflamación y dolor, causando fascitis plantar $^{6,7}$ Estos cambios degenerativos que suceden en la fascia plantar pueden dar lugar a inflamación aguda y crónica, calcificaciones en el origen de la fascia plantar y formaciones de tracción ósea causando dolor en la planta del pie pi $^{6}$. Esta patología es una de las causas más comunes de dolor en la extremidad inferior produciendo un gran impacto en la calidad de vida. El dolor de la fascitis plantar se caracteriza por dolor en la en la planta del pie, concretamente en el la parte medial y/o en el talón ${ }^{8,9}$. La fascitis plantar se considera un trastorno multifactorial y varios factores de riesgo implican la evolución de esta enfermedad. Aunque la evidencia es limitada se han planteado varios factores de riesgo tanto intrínsecos como extrínsecos. Algunos de los factores de riesgo intrínsecos implican una movilidad limitada de la primera articulación metatarso falángica, rango de movimiento limitado en la dorsiflexión de tobillo, pies planos, la edad (el riesgo aumenta sobre todo en aquellas personas que superen los 40 años), tensión del grupo muscular posterior de la pierna, engrosamiento de la fascia, discrepancia de longitud entre ambas piernas, espolón calcáneo, disminución de la fuerza en la pantorrilla, excesiva pronación del pie y un aumento del índice de masa corporal (IMC). Además, Owens BD et al ${ }^{10}$ afirmaron que existe más probabilidad de sufrir fascitis plantar en el sexo femenino que en el sexo masculino. Los factores de riesgo intrínsecos son aspectos individuales, como los problemas biomecánicos, la edad y el índice de masa corporal (IMC), entre otros. Los factores de riesgo extrínseco son influencias externas a la persona, como tipo de trabajo, actividades recreativas y áreas donde las actividades se realizan. Estudios recientes, como los de Riddle et al ${ }^{12}$, Jelinek y Fox ${ }^{13}$ e Irving et $\mathrm{al}^{11}$, examinaron qué factores pueden influir en el desarrollo del dolor plantar en poblaciones adultas. Describieron la relación de factores o variables intrínsecas, sobre todo la obesidad, la edad y los problemas biomecánicos. ${ }^{13}$
Dada la estructura y función del pie, cualquier condición problemática en él puede tener un profundo impacto negativo en la calidad de vida y función del paciente/Los problemas de pie pueden tener un gran impacto negativo en la función y en la calidad de vida, y evaluar la presencia y consecuencias del dolor de pie y la discapacidad es esencial para la cuantificación del deterioro y la evaluación de tratamientos ${ }^{14,15}$. En los últimos 20 años, los cuestionarios y escalas han emergido como una importante forma de evaluar y monitorizar pacientes. Son utilizadas en todo el mundo en la práctica diaria y la investigación como una forma de cuantificar la percepción del paciente de la discapacidad, la salud, y la calidad de vida. Estos instrumentos son económicos, fáciles de usar, específicas y fiables ${ }^{15,16}$. Las cuestionarios y escalas proporcionan respuestas objetivas sobre el estado y la función que ayudan a los terapeutas, cirujanos e investigadores a seguir un progreso de los pacientes y determinar si el su estado ha cambiado. Este proceso de cuantificación externa ha sido progresivamente adoptado y refleja con precisión el estado de salud del paciente mediante una metodología de autoinforme. De esta manera, la comprensión de los terapeutas y los investigadores de cómo la función del paciente y los síntomas han cambiado, con el tiempo o en respuesta a una intervención, pueden asimilarse rápidamente. Este tipo de medidas obtenidas de los pacientes son aplicables para una amplia gama de estados, enfermedades y lesiones y ayuda a la gestión progresiva mediante el reconocimiento de los efectos sobre las capacidades del paciente. En consecuencia, se adoptan con mayor frecuencia como el protocolo estándar para la medición y la evaluación del estado funcional. ${ }^{16}$

Con la intención de evaluar el impacto de los problemas en la extremidad inferior se han desarrollado varias herramientas: la escala American Orthopedic Foot and Ankle Society (AOFAS $)^{17}$, el Foot Function Index (FFI) ${ }^{18}$, el Foot Health Status Questionnaire ${ }^{19}$, el Self-Administered Foot Evaluation Questionnaire, la escala Roles \& Maudlsey o el Manchester Foot Pain and Disability Index (MFPDI $)^{20}$. Todas estas herramientas nos sirven para poder cuantificar variables como el dolor, la discapacidad o la limitación funcional del paciente mediante la percepción de este. En este estudio utilizaremos la escala FFI-sp, en las cual podemos medir de forma cuantitativa la limitación a la actividad, discapacidad, dolor, limitación funcional. Los resultados obtenidos serán comparados según sexo, edad e IMC de la muestra obtenida para 
determinar si existe una relación entre estas variables con el dolor, la discapacidad, la limitación funcional y la apariencia personal.

\subsection{Descripción del Foot Function Index (FFI) (Figura 1)}

- Finalidad: Evaluar la salud relacionada con el pie y la calidad de vida. - Contenido: Preguntas para evaluar la función general del pie, la salud del pie y la calidad de vida. El FFI tiene 3 subescalas: dolor (9 preguntas), discapacidad (9 preguntas) y limitación de la actividad (5 preguntas). - Número de ítems: El original consta de 23 ítems repartidos en 3 subescalas. • Opciones de respuesta: En el original, por cada ítem hay una escala visual analógica dividida en 10 segmentos iguales, con un rango de 0-9. Para completar el test, los pacientes tienen que puntuar cada ítem en la escala visual, siendo 0 la menor puntuación y 9 la máxima puntuación. Además, tienes que señalar el número de días de la última semana, en los cuales tuviste dolor de pie, siendo 0 la menor puntuación y 7 la máxima puntuación. En la adaptación al español (FFI-sp) hay un modelo en el que se exponen 3 opciones de respuesta en un rango de 1-3, siendo 1 la mínima puntuación y 3 la máxima puntuación. - Periodo específico de los ítems: 1 semana.

- Fines lucrativos: ninguno.

\subsubsection{Aplicación práctica}

- Como obtener: Disponible en la publicación original.

- Método de cumplimentación: Autoadministración o entrevistas.

- Interpretación de resultados: En un rango de $0-100 \%$, que se obtiene de la ecuación $(\mathrm{X} / 207)$ x 100 en la que $X$ es la suma de la puntuación de todos los ítems. Los puntajes más altos indican un empeoramiento de la salud de los pies y una peor calidad de vida. En el FFI-sp se sumarían las puntuaciones de cada ítem, siendo 23 la mínima puntuación y 69 la máxima puntuación. - Tiempo de cumplimentación: menos de 30 minutos.

- Carga de estudio: el que se dedique a la realización de tu propio estudio.

Figura 1. Foot Functional Index en español

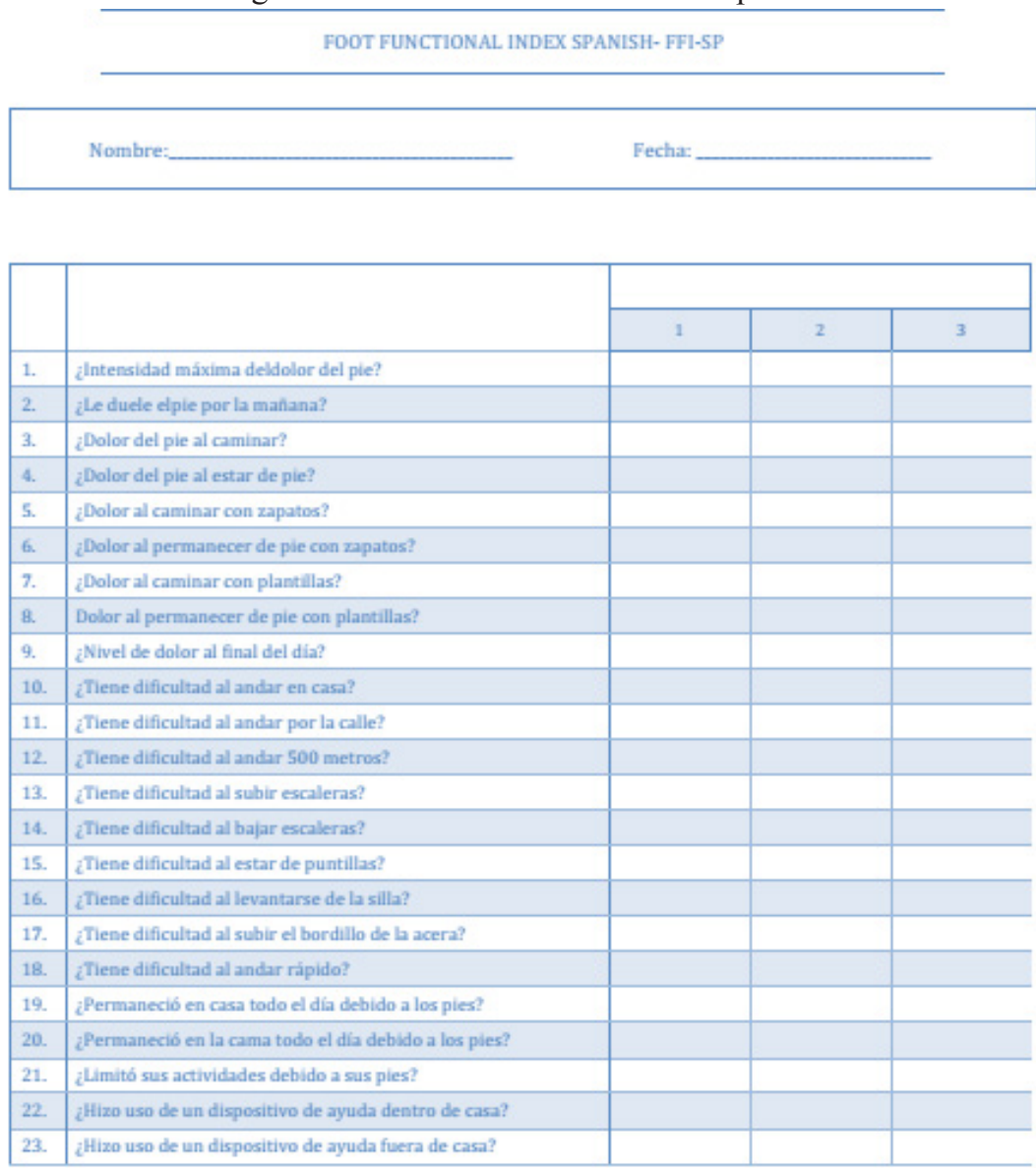




\subsubsection{Información psicométrica}

- Método de desarrollo: Adaptado de la información obtenida de encuestas anteriores, grupos de enfoque de pacientes y especialistas de pie. - Aceptabilidad: El cuestionario está escrito para un nivel de lectura de octavo grado en EEUU, el equivalente a $2^{\circ}$ de la ESO en España. - Fiabilidad: Los encuestadores de FFI reportaron que la FFI tenía una alta fiabilidad test-retest, con un coeficiente de correlación intraclase $(\mathrm{CCI})$ de 0,87 para el cuestionario completo. El CCI para las subescalas fue 0,69 , 0,81 y 0,84 para las subescalas de dolor, discapacidad y limitación de actividad, respectivamente. Budiman-Mak et al informaron el alfa de FFI Cronbach como 0,96 para el cuestionario completo, con la subescala alfa de 0,73 ,
0,93 y 0,95 para las subescalas de limitación de actividad, discapacidad y dolor, respectivamente, indicando alto nivel de confianza interna. En la adaptación al español se obtuvo una alfa de Cronbach del 0,965 para toda la escala y $0,95,0,96$ y 0,69 para las subescalas de dolor, discapacidad y limitación en la actividad respectivamente. Además, el FFI-sp tiene una correlación positiva moderada con el VAS, mientras más elevado es el FFI-sp más elevado será la puntuación en el VAS. Con el EuroQoL, SF-12 Physical, FHSQ Foot function, FHSQ QFH observamos una correlación negativa moderada y negativa alta con el FHSQ Foot pain, esto quiere decir que mientras más alto sea la puntuación en el FFI-sp más baja será la puntuación en el resto, una correlación inversa (Tabla 1)

Tabla 1. Contenido del cuestionario de salud del pie reportado por el paciente

\begin{tabular}{|c|c|c|c|c|c|c|c|c|}
\hline & $\begin{array}{c}\text { Dolor del } \\
\text { pie }\end{array}$ & $\begin{array}{c}\text { Salud } \\
\text { del pie }\end{array}$ & $\begin{array}{c}\text { Función } \\
\text { del pie }\end{array}$ & $\begin{array}{c}\text { Limitación } \\
\text { funcional }\end{array}$ & Autopercepción & $\begin{array}{c}\text { Aspecto } \\
\text { psicológico }\end{array}$ & Social & Ortopedia/zapatos \\
\hline FFI & $\mathrm{SI}$ & - & - & $\mathrm{Si}$ & - & $\mathrm{Si}$ & $\mathrm{SI}$ & $\mathrm{SI}$ \\
\hline
\end{tabular}

FFI: Foot Foot Posture Index

\section{Objetivos}

Determinar la relación entre las subescalas incluidas en el FFI-sp con el sexo, edad e IMC en pacientes con fascitis plantar.

\section{Material y metodos}

\subsection{Muestra}

La recogida de datos para este estudio se realizó en el Hospital Reina Sofía de Murcia, Hos- pital Morales Meseguer de Murcia y en la clínica Gallomur de Javalí Nuevo (Murcia). En sendos lugares se nos facilitó una sala acondicionada para llevar a cabo la recogida de datos. Esta se realizó entre el 14 de febrero y el 8 de marzo de 2017.

En este estudio se incluyen 28 sujetos. Los pacientes eran adultos, 13 hombres (46,4\%) y 15 mujeres $(53,6 \%)$ con una edad media de 54,18 años (SD 9,85) y una media de IMC de 28,76 (SD 4,2) que fueron diagnosticados por un traumatólogo con fascitis plantar crónica (tabla 2 y 3 ).

Tabla 2. Frecuencia de sexo de la muestra

\begin{tabular}{|l|c|c|}
\hline \multicolumn{1}{|c|}{ Sexo } & Frecuencia & Porcentaje \\
\hline Masculino & 13 & $46,4 \%$ \\
\hline Femenino & 15 & $53,6 \%$ \\
\hline Total & 28 & 100 \\
\hline
\end{tabular}

Tabla 3. Edad e IMC. Media y SD

\begin{tabular}{|l|l|l|}
\hline & Media & SD \\
\hline Edad & 54,18 & 9,85 \\
\hline IMC & 28,76 & 4,2 \\
\hline
\end{tabular}




\subsection{Criterios de inclusión y exclusión}

Los criterios de inclusión fueron: diagnóstico de fascitis plantar crónica ( $>3$ meses) unilateral o bilateral, ser mayor de 18 años. Los criterios de exclusión fueron: deterioro cognitivo (problemas para rellenar el cuestionario de forma autónoma o entrevista), sufrir otra enfermedad en el miembro inferior diferente a la fascitis plantar y haber tenido una intervención quirúrgica en los últimos 3 meses.

\subsection{Procedimiento}

Se obtuvo el consentimiento informado por parte de todos los participantes individuales incluidos en este estudio. El consentimiento informado fue elaborado por el departamento de fisioterapia de la Universidad de Murcia. Los pacientes entraron de uno en uno a la sala donde se realiza la recogida de datos. Se les facilitó el consentimiento informado y tras esto se les facilitaron ambos cuestionarios para cumplimentarlos. Se explicó cómo rellenar ambos cuestionarios y se respondieron a las dudas que surgieron mientras los cumplimentaron. El tiempo medio aproximado de cumplimentación de cada paciente del FFI fue de 5 a 15 minutos.

\subsection{Análisis estadístico}

Tras obtener todos los datos de los pacientes incluidos en el estudio creamos una base de datos con el fin de observar si existen cambios significativos en el dolor, discapacidad y limitación funcional en relación con el sexo, la edad y el IMC de nuestra muestra. Para ello sacamos el resultado medio obtenido en cada una de las subescalas del FFI-sp y las relacionamos mediante la prueba t-student para muestras independientes. Además, se realizó la prueba de Shapiro-Wilk, que se utiliza para muestras menores de 30 individuos para analizar si la muestra procede de una distribución normal. El análisis estadístico fue hecho por el IBM SPSS Statistics (Versión 19).

\section{Resultados (ver tabla 4)}

Tanto la limitación a la actividad como la apariencia personal son dos aspectos que se cuantifican en el FFI-sp. Debido a los problemas a la hora de contestar e interpretar estas cuestiones por parte de los pacientes se eliminaron del estudio y por lo tanto del análisis estadístico. Para observar si existen cambios en nuestra muestra hemos comparado los resultados obtenidos en las diferentes subescalas dividiendo la muestra $(n=28)$ según sexo (masculino y femenino), edad $(<54,18$ y $>54,18)$ e IMC $(<28,7621$ y $>28,7621)$. En las pruebas de Shapiro-Wilk hemos obtenido valores por encima del 0,05 por lo que nuestros datos proceden de una distribución normal.

\subsection{Comparación de dolor, discapacidad y limitación funcional según sexo}

No hay diferencias estadísticamente significativas entre las variables Subescala de dolor del FFI-sp ( $p$-valor $=0,198)$, Subescala de dolor del MFPDI ( $\mathrm{p}$-valor $=0,794)$, Subescala de discapacidad del FFI-sp (p-valor $=0,916)$ y Subescala de limitación funcional ( $\mathrm{p}$-valor $=$ $0,906)$ en relación a la variable sexo.

\subsection{Comparación de dolor, discapacidad y limitación funcional según edad}

No hay diferencias estadísticamente significativas entre las variables Subescala de dolor del FFI-sp ( $p$-valor $=0,946)$, Subescala de dolor del MFPDI ( $\mathrm{p}$-valor $=0,268$ ), Subescala de discapacidad del FFI-sp $(\mathrm{p}$-valor $=0,735)$ y Subescala de limitación funcional ( $\mathrm{p}$-valor $=$ 0,741 ) en relación a la variable edad.

\subsection{Comparación de dolor, discapacidad y limitación funcional según IMC}

No hay diferencias estadísticamente significativas entre las variables Subescala de dolor del FFI-sp ( $p$-valor $=0,190)$ y Subescala de discapacidad del FFI-sp ( $\mathrm{p}$-valor $=0,916)$ en relación a la variable IMC.

Tabla 4. Resultados

\begin{tabular}{|l|l|l|l|}
\hline t-student (p-valor) & Sexo & Edad & IMC \\
\hline Subescala del dolor FFI & 0,185 & 0,946 & 0,116 \\
\hline Subescala de discapacidad FFI & 0,916 & 0,735 & 0,330 \\
\hline
\end{tabular}




\section{Discusión}

\subsection{Población de estudio}

Partimos de una muestra de 28 individuos con un rango de edad de 35 a 68 años, con una media de edad de 54,18 con una desviación típica de 9,85 . Owens BD et $a{ }^{10}$ señalan en su estudio que el incremento de la edad es un factor de riesgo, incrementándose sobre todo a partir de los 40 años, donde el riesgo de padecer fascitis plantar aumentaba. En nuestra muestra hemos obtenido una edad media de 54,18 años y solo 1 individuo por debajo de los 40 años, por lo que se corresponde a lo citado por estos autores. En cuanto al sexo, nos encontramos ante una muestra bastante homogénea, donde predomina el sexo femenino $(53,6 \%)$ frente al sexo masculino $(46,4 \%)$. Owens BD et $\mathrm{al}^{10} \mathrm{e}$ Irving $\mathrm{DB}$ et $\mathrm{al}^{11}$ indican que existe una mayor probabilidad de tener fascitis plantar en el sexo femenino. Respecto al IMC, en nuestra muestra hemos encontrado una media de 28,76, datos que indican sobrepeso. Autores como Yanbin $\mathrm{X}$ et $\mathrm{al}^{2}$, Irving DB et $\mathrm{al}^{11}$, Riddle et $\mathrm{al}^{12}$ y Jelinek HF y Fox $\mathrm{D}^{13}$ Ortega- Ávila et $\mathrm{al}^{21}$ determinaron que un IMC alto es un factor de riesgo para sufrir fascitis plantar, por lo que lo que señalaban en su estudio concuerda con los datos obtenidos de nuestra muestra. Sin embargo, excepto Irving et $\mathrm{al}^{11}$ Riddle et $\mathrm{al}^{12}$ y Jelinek HF y Fox $\mathrm{D}^{13}$ Ortega-Ávila et $\mathrm{al}^{21}$, todos estos autores llevaron a cabo estudios diferentes al que yo he realizado. Estos autores también recogen como factores de riesgo pies planos, excesiva pronación del pie y limitación de la dorsiflexión de tobillo. A pesar de ello, como hemos comentado en la introducción, en términos de evidencia, la influencia de estos factores (sexo, edad e IMC) sobre el desarrollo del dolor es limitada.

\subsection{Relación de variables con la fascitis plantar}

Una revisión de la literatura muestra que hay una ausencia de estudios que determinen la contribución y la relación entre variables clínicas como el sexo, la edad y el IMC con las subescalas correspondientes del FFI-sp. Este estudio verifica la existencia de la relación y la influencia entre las citadas variables clínicas con las subescalas correspondientes del FFI-sp. En estudios similares la variable con mayor relación con la fascitis plantar es el IMC. Riddle DL et al ${ }^{12}$ comparó una serie de variables entre las que se incluyen el sexo, la edad y el IMC asociadas a la discapacidad causada por la fascitis plantar. La discapacidad fue cuantificada por la Lower Extremity Functional Scale (LEFS), una medida validada de auto-reporte de la discapacidad. La población de este estudio era $\mathrm{n}=50$ de los cuales un $34 \%$ eran hombres y un $66 \%$ mujeres, un $80 \%$ estaba por encima de 25 en el IMC y un $78 \%$ de los pacientes tenía una edad superior a los 40 años. Respecto a la población de estudio podríamos decir que existen similitudes. Hay mayor predominio de mujeres $(66 \%)$ al igual que en nuestra muestra, con un 52,9\%, aunque en nuestro caso no podamos asegurarlo con totalidad, ya que este predominio puede deberse a la aleatoriedad debido a lo pequeña que es nuestra muestra. El IMC es similar, ya que afirma que el $80 \%$ de los pacientes tenía un IMC superior al 25 y en nuestra muestra hemos obtenido una media del IMC de 28,76 con una desviación típica de 4,2. En cuanto a los resultados la única relación que se obtuvo fue entre la variable IMC con el grado de discapacidad reportado por la LEFS con un $\mathrm{p}$-valor $=0,003$, valor similar a la relación del IMC con el dolor y la limitación funcional reportado por el MFPDI con un $\mathrm{p}$-valor $=0,013$ $\mathrm{y}$ un $\mathrm{p}$-valor $=0,03$ respectivamente. OrtegaÁvila et $\mathrm{al}^{21}$ llevaron a cabo un estudio similar al que Jelinek HF y Fox $\mathrm{D}^{13}$ e Irving DB et $\mathrm{al}^{22}$ evaluando el impacto en la calidad de vida que produce el dolor de pie reportado por el FHQS. Estos tres estudios son los más similares al que nosotros hemos llevado a cabo, junto al de Riddle DL et $\mathrm{al}^{12}$, ya que evalúan la relación entre distintas variables clínicas en pacientes con dolor de pie mediante cuestionarios. En este caso, Ortega-Ávila et al utilizaron una muestra de 62 participantes compuesta por 25 hombres $(40,3 \%)$ y 37 mujeres $(59,7 \%)$ con una media del IMC de 26,29 (inferior a la obtenida en nuestro estudio de 28,76 ) con una desviación típica de 3,79 . Se encuentra mayor relación entre la subescala de salud del pie del FHSQ y el IMC con pvalor $=0,001$, además también se encuentra relación con la edad. En la conclusión de este estudio incluye al IMC y la edad con una de las variables a conocer en el manejo clínico. Este estudio es el único encontrado en el que se determina que la variable edad tiene relación con pacientes con dolor de pie, por lo que esta afirmación carece de suficiente criterio de consistencia. 


\subsection{Limitaciones del estudio}

Hay que aclarar que este estudio tiene limitaciones debido a que nuestra muestra es demasiado pequeña y se necesita de una muestra más grande para que corroborar con más seguridad los resultados obtenidos. Otra de las limitaciones es la falta de literatura en lo referido a este tema, ya que hay pocas publicaciones de estudios que indiquen una relación entre el sexo, la edad y el IMC con la salud del pie, reportada por cuestionarios.

\section{Conclusión}

No se encontró relación entre las subescalas incluidas en el FFI-sp y el IMC. No se encontraron relaciones entre el sexo y edad y las subescalas incluidas en el FFI-sp.

\section{Bibliografía}

1. Kamonseki DH, Goncalves GA, Yi LC, Junior IL. Effect of stretching with and without muscle strengthening exercises for the foot and hip in patients with plantar fasciitis: A randomized controlled single-blind clinical trial. Man Ther. 2016;23:76-82. 2. Yanbin X, Haikun C, Xiaofeng J, Wanshan Y, Shuangping L. Treatment of Chronic Plantar Fasciitis With Percutaneous Latticed Plantar Fasciotomy. The Journal of Foot and Ankle Surgery. 2015;54 (5):856-9. 3. Zhou B, Zhou Y, Tao X, Yuan C, Tang K. Classification of Calcaneal Spurs and Their Relationship With Plantar Fasciitis. J Foot Ankle Surg. 2015;54 (4):594-600. 4. Mardani-Kivi M, Mobarakeh MK, Hassanzadeh Z, Mirbolook A, Asadi K, Ettehad H, et al. Treatment outcomes of corticosteroid injection and extracorporeal shock wave therapy as two primary therapeutic methods for acute plantar fasciitis: a prospective randomized clinical trial. The Journal of Foot and Ankle Surgery. 2015;54 (6):1047-52.

5. Ajimsha M, Binsu D, Chithra S. Effectiveness of myofascial release in the management of plantar heel pain: a randomized controlled trial. The Foot. 2014;24 (2):66-71. 6. Puttaswamaiah Rea. Degenerative plantar fasciitis: A review of current concepts. The Foot. 2007 (1):3.

7. Werner RA, Gell N, Hartigan A, Wiggerman N, Keyserling WM. Risk factors for plantar fasciitis among assembly plant workers. PM R. 2010;2 (2):110-6

8. Arslan A, Koca TT, Utkan A, Sevimli R, Akel I. Treatment of Chronic Plantar Heel Pain With Radiofrequency Neural Ablation of the First Branch of the Lateral Plantar Nerve and Medial Calcaneal Nerve Branches. J Foot Ankle Surg. 2016;55 (4):767-71. 9. Cotchett MP, Whittaker G, Erbas B. Psychological variables associated with foot function and foot pain in patients with plantar heel pain. Clin Rheumatol. 2015;34 (5):957-64. 10. Owens BD, Wolf JM, Seelig AD, Jacobson IG, Boyko EJ, Smith B, et al. Risk Factors for Lower Extremity Tendinopathies in Military Personnel. Orthop J Sports Med. 2013;1 (1):2325967113492707.

11. Irving DB, Cook JL, Young MA, Menz HB. Obesity and pronated foot type may increase the risk of chronic plantar heel pain: a matched case-control study. BMC Musculoskelet Disord. 2007;8:41.

12. Riddle DL, Pulisic M, Sparrow K. Impact of demographic and impairment related variables on disability associated with plantar fasciitis. Foot Ankle Int. 2004;25 (5):311-7. 13. Jelinek HF, Fox D. Foot Health and Elevated Body Mass Index. The Foot and Ankle Online Journal. 2009;2 (8):4.

14. Gijon-Nogueron G, Ndosi M, Luque-Suarez A, Alcacer-Pitarch B, Munuera PV, Garrow A, et al. Crosscultural adaptation and validation of the Manchester Foot Pain and Disability Index into Spanish. Qual Life Res. 2014;23 (2):571-9. 15. González-Sánchez M, Velasco-Ramos E, Ruiz-Muñoz M, CuestaVargas AI. Cross-cultural adaptation and validation of the Spanish version of the American Academy of Orthopaedic Surgeons-Foot and Ankle Module (AAOS-FAMsp). J Orthop Surg Res. 2016;11 (1):74.

16. Cuesta-Vargas AI, Gabel CP. Validation of a Spanish version of the Spine Functional Index. Health Qual Life Outcomes. 2014;12:96.

17. Kitaoka HB, Alexander IJ, Adelaar RS, Nunley JA, Myerson MS, Sanders M. Clinical rating systems for the ankle-hindfoot, midfoot, hallux, and lesser toes. Foot Ankle Int. 1994;15 (7):349-53.

18. Budiman-Mak E, Conrad KJ, Roach KE. The Foot Function Index: a measure of foot pain and disability. J Clin Epidemiol. 1991;44 (6):561-70.

19. Bennett PJ, Patterson C, Wearing S, Baglioni T. Development and validation of a questionnaire designed to measure foot-health status. J Am Podiatr Med Assoc. 1998;88 (9):419-28. 
20. Garrow AP, Papageorgiou AC, Silman AJ, Thomas E, Jayson MI, Macfarlane GJ. Development and validation of a questionnaire to assess disabling foot pain. Pain. 2000;85 (1-2):107-13.

21. Ortega-Avila AB, Cuesta-Vargas AI, Jiménez-Cebrián AM, Labajos Manzanares MT, Barón-López FJ, Bennet P. Contribution Levels of Intrinsic Risk Factors to the Management of Patients with Plantar Heel Pain A Pilot Study. J Am Podiatr Med Assoc. 2016;106 (2):88-92.

22. Irving DB, Cook JL, Young MA, Menz HB. Impact of chronic plantar heel pain on health-related quality of life. J Am Podiatr Med Assoc. 2008;98 (4):283-9. 